\title{
On the bias and stability of the results of comparative judgment
}

\author{
Elise A. V. Crompvoets ${ }^{1,2}$, Anton A. Béguin ${ }^{3}$, Klaas Sijtsma ${ }^{1}$ \\ ${ }^{1}$ Tilburg University, the Netherlands \\ ${ }^{2}$ Cito, the Netherlands \\ ${ }^{3}$ International Baccalaureate, United Kingdom
}

Corresponding Author:

Elise Crompvoets. E-mail: e.a.v.crompvoets@uvt.nl. Phone: 0031134664328. Institutional address Tilburg University: P.O. Box 90153, 5000 LE Tilburg, The Netherlands. 


\title{
STABILITY OF COMPARATIVE JUDGMENT RESULTS
}

\begin{abstract}
Comparative judgment is a method that allows measurement of a competence by comparison of items with other items. In educational measurement, where comparative judgment is becoming an increasingly popular assessment method, items are mostly students' responses to an assignment or an examination. For assessments using comparative judgment, the Scale Separation Reliability (SSR) is used to estimate the reliability of the measurement. Previous research has shown that the SSR may overestimate reliability when the pairs to be compared are selected with certain adaptive algorithms, when raters use different underlying models/truths, or when the true variance of the item parameters is below one. This research investigated bias and stability of the components of the SSR in relation to the number of comparisons per item to increase understanding of the SSR. We showed that many comparisons are required to obtain an accurate estimate of the item variance, but that the SSR can be useful even when the variance of the items is overestimated. Lastly, we recommend adjusting the general guideline for the required number of comparisons per item to 41 comparisons per item. This recommendation partly depends on the number of items and the true variance in our simulation study and needs further investigation.
\end{abstract}

Keywords: bias, comparative judgment, pairwise comparison, reliability, stability 


\section{STABILITY OF COMPARATIVE JUDGMENT RESULTS}

\section{On the bias and stability of the results of comparative judgment}

Comparative judgment is a method that allows measurement of a competence by comparison of items. When items are compared in pairs, comparative judgment is also known as pairwise comparison. This method has been used in different contexts ranging from sports to marketing to educational assessment, with different models for each context (e.g., Agresti, 1992; Böckenholt, 2001; 2006; Brinkhuis, 2014; Cattelan, 2012; Maydeu-Olivares, 2002; MaydeuOlivares \& Böckenholt, 2005; Stark, 2011). In educational measurement, where comparative judgment is becoming an increasingly popular assessment method (Bramley \& Vitello, 2018; Lesterhuis et al., 2017), items are mostly students' responses to an assignment or an examination. The assignment or the examination is used to measure a competence of the students, and the students' responses give an indication of their competence level. The method has been used in a variety of contexts, ranging from art assignments (Newhouse, 2014) to academic writing (Van Daal et al., 2016) and mathematical problem solving (Jones \& Alcock, 2013). These contexts have in common that the competencies are difficult to disentangle into sub-aspects together defining the competencies. Therefore, they are difficult to measure validly using analytical scoring schemes such as rubrics or criteria lists (Van Daal et al., 2016), which are conventional measurement methods used in education. In contrast to these analytic measurement methods, which assume that a competence can be operationalized by means of a list of sub-aspects and evaluate each aspect separately, comparative judgment is a holistic measurement method where a competence is evaluated as a whole (Pollitt, 2012); simply asking which of two items scores higher on the competence of interest suffices.

For complex competencies like art assignments, academic writing, and mathematical problem solving, it is possible that a higher validity can be obtained using comparative judgment 


\section{STABILITY OF COMPARATIVE JUDGMENT RESULTS}

instead of rubrics or criteria lists (Pollitt, 2012; Van Daal, 2016) because of its holistic character and the greater possibility of raters to use their expertise in their judgments compared to rubrics or criteria lists. In addition to the claim of higher validity of comparative judgment, Pollitt (2012) claimed that comparative judgment also results in higher reliability compared to using rubrics or criteria lists. However, later research has shown that this claim is likely to be too optimistic (e.g., Bramley, 2015; Bramley \& Vitello, 2018; Crompvoets, Béguin, \& Sijtsma, 2020; 2021).

To explain why Pollitt's (2012) claim is too optimistic, we first define two types of reliability in the context of comparative judgment: the benchmark reliability (Crompvoets et al., 2020, 2021) and the Scale Separation Reliability (SSR; e.g., Bramley, 2015; Crompvoets et al., 2020). Both forms of reliability are based on parameters of the Bradley-Terry-Luce (BTL; Bradley \& Terry, 1952; Luce, 1959) model. This model is defined as follows. Let $K$ be the number of items, let $i$ and $j(i, j=1, \ldots, K)$ be item indices, and let $\theta_{i}$ and $\theta_{j}$ be the parameters

of items $i$ and $j$. Furthermore, let $X_{i j}$ be the outcome of the inter-item comparison where $X_{i j}=1$ means that item $i$ was preferred to item $j$, and $X_{i j}=0$ means that item $j$ was preferred to item $i$. The BTL model defines the probability that item $i$ is preferred to item $j$ in a paired comparison by means of

$$
P\left(X_{i j}=1 \mid \theta_{i}, \theta_{j}\right)=\frac{\exp \left(\theta_{i}-\theta_{j}\right)}{1+\exp \left(\theta_{i}-\theta_{j}\right)}
$$

We interpret $\theta$ as an item parameter, but we may also interpret it as a person parameter for the competence of one person. Thus, items and persons are not clearly distinguished in the BTL model for comparative judgment.

The benchmark reliability is only known in simulated data and is computed as the squared correlation between the true (simulated) item parameters and the item parameter 


\section{STABILITY OF COMPARATIVE JUDGMENT RESULTS}

estimates. Let $\theta$ be the item parameter in the generating model and let $\hat{\theta}$ be the item parameter estimate. The benchmark reliability can then be computed as

$$
\rho_{\widehat{\theta} \widehat{\theta},}=\operatorname{cor}(\theta, \widehat{\theta})^{2}
$$

This definition of reliability corresponds with the definition of reliability as $\rho^{2}(\theta, \hat{\theta})$ in classical test theory (Lord \& Novick, 1968), where $\theta$ represents the true score and $\hat{\theta}$ represents the observable test score. Since we are interested in reliability of the measurement of a specific set of items, benchmark reliability is used as the true reliability of this set of items.

The SSR is an estimate of reliability that is based on the Index of Subject Separation formulated by Andrich and Douglas (1977, as cited in Gustafsson, 1977) and is computed as follows. We assume that items are compared in pairs and that the location parameters of these items on the latent competence scale are of interest. Let $S^{2}(\theta)$ be the estimated true variance of the object parameters and let $S^{2}(\hat{\theta})$ be the variance of the estimated object parameters. Furthermore, let $M S E$ be the mean of the squared standard errors corresponding to the item parameter estimates, computed as

$$
M S E=\frac{1}{K} \sum_{i}^{K} S E\left(\widehat{\theta}_{i}\right)^{2}
$$

The SSR can then be written as

$$
S S R=\frac{S^{2}(\theta)}{S^{2}(\widehat{\theta})}
$$

where

$$
S^{2}(\theta)=S^{2}(\hat{\theta})-M S E
$$

that is, the observed variance minus an error term (Bramley, 2015).

Research (Bramley, 2015; Bramley \& Vitello, 2018; Crompvoets et al., 2020) has shown that the SSR might overestimate reliability [Equation (2)] in certain situations. These include the 


\section{STABILITY OF COMPARATIVE JUDGMENT RESULTS}

use of certain adaptive algorithms to select the pairs that raters have to compare. Pollitt's (2012) claim that comparative judgment results in higher reliability than using rubrics or criteria lists is based on a study using an adaptive algorithm to select the pairs that are compared in combination with the SSR. Other situations in which the SSR may overestimate benchmark reliability are when raters behave inconsistent amongst each other, which would be reflected in the BTL model by different parameters for the same items, and perhaps when the true variance of the item parameters is below 1 as well (Crompvoets et al., 2021). The result that the SSR may overestimate reliability suggests why Pollitt's (2012) claim that comparative judgment results in higher reliability is likely too optimistic. Moreover, the result that the SSR may overestimate reliability is problematic because (1) reliability estimates should provide a lower bound to reliability to avoid reporting reliability that is too high and therefore promises too much (Hunt \& Bentler, 2016; Sijtsma, 2009) and (2) most recommendations about the number of required comparisons are based on achieving at least a user-defined value of the SSR (e.g., Verhavert et al., 2019).

To the best of our knowledge, no one has thoroughly investigated and reported the positive bias of the SSR. Previous research that reported the bias of the SSR has stopped at the conclusion that the SSR was biased (Bramley, 2015; Bramley \& Vitello, 2018) or has only led to speculations about the meaning of the bias due to either adaptive pair selection (Crompvoets et al., 2020), different rater probabilities, or small true variances (Crompvoets et al., 2021).

One might reason that the behavior of the SSR needs no investigation, because its value can easily be derived from the two components $S^{2}(\hat{\theta})$ and $M S E$ [Equation (3)]. However, understanding which factors influence the value of the SSR is complex. The easy way to investigate a measure like the SSR would be to vary only one component and keep the other 


\section{STABILITY OF COMPARATIVE JUDGMENT RESULTS}

components constant to see how the value of the measure changes with the value of the

component. However, both $S^{2}(\hat{\theta})$ and $M S E$ are based on the parameter estimates $\widehat{\boldsymbol{\theta}}$ from the underlying model. This means that a shift in the item parameters affects both components simultaneously, which renders varying one component while the other is kept constant unrealistic. Another complication is that all item parameter estimates are mutually dependent because we estimate the parameters based on comparisons of the items with each other. This means that every additional comparison changes all item parameter estimates, so we cannot vary one item parameter estimate keeping the other item parameter estimates constant. Moreover, the changes of item parameter estimates after one comparison depend on the parameters of the items that are compared; the outcome of the comparison, which is not always straightforward because we use a probabilistic model; the total number of items and their parameters; and the outcomes of all previous comparisons, which is not always straightforward due to the use of a probabilistic (e.g., BTL) model. Therefore, it is highly relevant to investigate the behavior of the SSR.

Because all quantities needed to estimate the SSR [Equation (3)] are based on the parameter estimates $\widehat{\boldsymbol{\theta}}$ from the underlying model, this study focused on the parameter estimates used in the computation of the SSR. Specifically, we investigate the bias and stability of the parameter estimates. We defined these outcomes in the Method section. Because parameter estimates depend on the amount of data available, we investigated bias and stability of the parameter estimates in relation to the number of comparisons.

The goal of this study was to gain insight into the bias and stability of the parameter estimates and the SSR of comparative judgment in educational measurement from two perspectives. In addition, we aimed to use this information either to support the guideline about the number of required comparisons per item from Verhavert et al. (2019) or to provide a new 


\section{STABILITY OF COMPARATIVE JUDGMENT RESULTS}

guideline based on the results from this study. First, we adapted the guideline for the required number of observations to obtain stable results for the one-parameter item response model or Rasch model (Rasch, 1960) for regular multiple choice tests to the BTL model used for comparative judgment. Second, we investigated the bias and stability of the parameter estimates and SSR of comparative judgment in a simulation study. In the discussion, we will reflect on the two perspectives.

\section{Sample size guideline adaptation to the BTL model}

Most researchers and test institutions use experience to determine how many observations are required to obtain stable model parameter estimates. The numbers of observations used for regular multiple-choice tests in research and practice are coherent with the only guideline (Parshall et al., 1998) we found in the literature. The guideline states that for the one-parameter item response model, at least 200 observations per item are required to obtain stable item location parameter estimates. For the mathematically related (Andrich, 1978) BTL model, no guidelines exist that describe how many observations are required in educational measurement for obtaining stable item parameter estimates. In this section, we first describe how the Rasch model and the BTL model are related, and then adapt the guideline from the Rasch model to the BTL model. In the Discussion section, we will evaluate this guideline in relation to the outcomes of the simulation study from the next section and in relation to the literature.

The Rasch model is defined as follows. Let $N$ be the number of persons in the sample, let $i(i=1, \ldots, N)$ be the person index, and let $\theta_{i}^{*}$ be the parameter of person $i$ on the latent variable scale, where the * indicates that $\theta_{i}^{*}$ differs from $\theta_{i}$ used in the BTL model [Equation (1)]. Let $K$ be the number of items, let $j(j=1, \ldots, K)$ be the item index, and let $\beta_{j}$ be the parameter of item $j$ on the latent variable scale. Furthermore, let $X_{i j}$ be the outcome of the person-item comparison 


\section{STABILITY OF COMPARATIVE JUDGMENT RESULTS}

where $X_{i j}=1$ means that person $i$ answered item $j$ correctly, and $X_{i j}=0$ means that person $i$ answered item $j$ incorrectly. The Rasch model defines the probability that person $i$ answers item $j$ correctly by means of

$$
P\left(X_{i j}=1 \mid \theta_{i}^{*}, \beta_{j}\right)=\frac{\exp \left(\theta_{i}^{*}-\beta_{j}\right)}{1+\exp \left(\theta_{i}^{*}-\beta_{j}\right)}
$$

We note that although mathematically it would have made sense to use $\beta_{i}$ and $\beta_{j}$ in the formulation of the BTL model [Equation (1)] for equivalence with the Rasch model, we chose to follow the conventional notation of the BTL model in comparative judgment contexts using $\theta$ notation for the items.

Andrich (1978) showed that the Rasch model and the BTL model are equivalent. This means that a person-item comparison in the Rasch model is mathematically equivalent to an inter-item comparison in the BTL model. Therefore, it makes sense to adapt the guideline for the Rasch model about the required number of observations for stable model estimates to the BTL model.

Our starting point for the guideline adaptation is the item, since items are present in both the Rasch model and the BTL model. In addition, the guideline Parshall et al. (1998) suggested aims at obtaining stable item parameter estimates. We assume that the number of items in the test that the Rasch model analyzes is the same as the number of items in the set of paired comparisons that is analyzed by means of the BTL model. However, the manner in which we obtain additional observations for an item differs in two ways between the models. First, one observation for the Rasch model contains information about one item and one person, whereas one observation for the BTL model contains information about two items. Second, each observation for an item in the Rasch model is obtained from a person belonging to a population 


\section{STABILITY OF COMPARATIVE JUDGMENT RESULTS}

with many possible parameter values, whereas each observation for an item in the BTL model is obtained from an item in the fixed set of items under investigation. Therefore, for the BTL model, the information obtained from one observation may depend on the item parameters in the set, which is different for the Rasch model, where the information also depends on the sample of persons.

There are two ways to adapt the guideline from the Rasch model for use with the BTL model. The first adaptation is to equate the number of required observations per item for the BTL to the required number for the Rasch model; that is, 200 observations per item (Guideline 1). Since each comparative judgment/observation for the BTL model contains information about two items, this adaptation means that compared to the Rasch model, we need half of the total number of observations. We illustrate this with an example. Suppose we have 20 items in both models. The guideline of 200 observations per item for the Rasch model means that we need 200 (persons) $\times 20$ (items) $=4,000$ observations in total for a 20 -item test to obtain stable item parameter estimates. The adapted guideline of 200 observations per item for the BTL model means that we need 200 (comparisons per item) / 2 (items per comparison) $\times 20$ (items) $=$ 2000 observations in total for a 20 -item test to obtain stable item parameter estimates.

The second possibility is to equate the total number of observations for a set of items instead of one item. Using Parshall et al.'s guideline for the Rasch model, 4,000 observations are required for a set of 20 items to obtain stable item parameter estimates. Adapted to the BTL model following the second guideline, this would mean that 4,000 paired comparisons in total are required to get stable item parameter estimates, which would mean (4,000 comparisons $\times$ 2 items per comparison) / 20 items $=400$ observations per item. This is our Guideline 2 . This means that compared to the Rasch model, we need twice as many observations per item for 


\section{STABILITY OF COMPARATIVE JUDGMENT RESULTS}

stable item parameter estimates from the BTL model. This makes sense, because each observation in a comparative judgment setting contains information about two items, so only half of the information concerns each item. We will evaluate both guidelines in the discussion section of this paper. One should note that the current recommendations for the numbers of comparisons per item based on a meta-analysis of comparative judgment applications range from 12 to 37 (Verhavert et al., 2019), which shows a large discrepancy with both the 200 and 400 comparisons per item according to the two adapted guidelines.

Although the number of observations per item is stable across different numbers of items, the number of items influences which items are compared. The number of unique comparisons in a comparative judgment setting equals $K(K-1) / 2$, which is nonlinear in $K$. This means that the number of times each unique comparisons is made differs for different numbers of comparisons. Table 1 illustrates this: using guideline 2, for 20 items, all unique comparisons should be made 21 times (on average). On the other hand, for 1,000 items, all unique comparisons should be made 0.4 times, which means that not even all unique comparisons are made.

\section{Bias and stability of SSR reliability estimate components}

We investigated in a simulation study: (a) how many comparisons are required to obtain a stable and unbiased variance of the parameter estimates, $S^{2}(\hat{\theta})$; (b) how many comparisons are required to obtain a coverage of $95 \%$-confidence intervals for the parameter estimates $\widehat{\boldsymbol{\theta}}$ using the standard errors SE( $(\widehat{\boldsymbol{\theta}})$ of $95 \%$; and (c) how the SSR develops with increasing number of comparisons. We investigated these outcomes in situations in which we expected the SSR to underestimate benchmark reliability, because it is easier to understand the SSR and its components in these situations than in situations where we do not know why the SSR 


\section{STABILITY OF COMPARATIVE JUDGMENT RESULTS}

overestimates benchmark reliability. The R-code of the simulation study is available at https://osf.io/x7qzc/.

\section{Method}

Simulation set-up. The simulation design had two factors. First, we varied the number of items $N=\{20,30,50,100\}$ to investigate whether the number of items affects the stability of the SSR estimate. Second, we used five different variances of the simulated item parameters. In the first condition, we used a variance of zero, which means that all items had the same location on the scale. We used this condition as a benchmark to investigate when the SSR was stable at zero, because the SSR should be zero if the true variance is zero, see [Equation (3)]. In the second condition, we used a variance of 1.59 , which is a realistic value based on the argumentative writing dataset 'having children' used in Van Daal (2020, data retrieved from https://osf.io/wpbhk/?view_only=7aa609162ca146bbbbe9236c9224b668). Argumentative writing refers to one's ability to express, argue for, and refute objections of one's opinion about a specific topic (Van Daal, 2020, p. 175). This dataset contained 1,224 comparative judgments performed by 55 raters of 135 texts written by students in the fifth year of secondary education on the topic 'having children'. Compared with the summary of several datasets in the metaanalysis of Verhavert et al. (2019), we argue that this dataset is realistic and representative of datasets obtained using comparative judgment for educational measurement. Furthermore, we added the variance conditions $0.5,1$, and 3 to obtain information about the results in between and beyond the benchmark variance and the realistic variance.

For each of the 4 (Number of Items) x 5 (Variance of Items) = 20 design conditions, we repeated the same procedure 100 times. We first selected to item pairs $(1,2),(2,3),(3,4)$, et cetera, until $(K-1, K)$ and $(K, 1)$ to create a linked comparison design. For each item pair, we 


\section{STABILITY OF COMPARATIVE JUDGMENT RESULTS}

simulated a comparison in which the probability of preferring one item to the other was given by the BTL model [Equation (1)]. After these $K$ comparisons, we estimated the BTL model (using the open source R-code from Crompvoets et al., 2020) and computed $S^{2}(\hat{\theta})$ and the SSR based on the parameter estimates from the BTL model for the first time. Subsequently, we compared a randomly selected pair of items, estimated the BTL model parameters, and computed $S^{2}(\hat{\theta})$ and the SSR after each comparison until the maximum number of comparisons of 200 per item was reached. Lastly, we computed the number of comparisons per item required to obtain a stable variance of the parameter estimates $S^{2}(\hat{\theta})$ at the true parameter variance and the number of comparisons per item required to obtain a correct coverage of the $95 \%$ confidence interval for the parameter estimates $\widehat{\boldsymbol{\theta}}$.

We determined the number of comparisons per item required for a stable and accurate estimate to be the number of comparisons where $12 \mathrm{~K}$ subsequent comparisons produced a value within a range around the true value, both for $S^{2}(\hat{\theta})$ and for the coverage of the $95 \%$ confidence intervals. The range of accurate values was defined as the range between 1 standard error below the true value and 1 standard error above the true value. We based the $12 \mathrm{~K}$ subsequent comparisons on the guideline of 12 comparisons per item from the meta-analysis of Verhavert et al. (2018).

\section{Results}

Figure 1 shows the development of $S^{2}(\hat{\theta})$ (top row), $M S E[S E(\widehat{\theta})]$ (middle row), and the SSR (bottom row) with increasing numbers of comparisons per item of each of the 100 simulations per design cell and of the average for all true variance conditions for 50 items. On average, $S^{2}(\hat{\theta})$ seems to converge to the true variance, but not for every single simulated data set. Comparing the top- and middle rows, we see that there is much more variation in $S^{2}(\hat{\theta})$ than 


\section{STABILITY OF COMPARATIVE JUDGMENT RESULTS}

in $\operatorname{MSE}[S E(\widehat{\theta})]$ across simulations. The variation in development across simulations of both $S^{2}(\hat{\theta})$ and $\operatorname{MSE}[S E(\hat{\theta})]$ was larger for larger true variance values. Interestingly, although $S^{2}(\hat{\theta})$ and $\operatorname{MSE}[\operatorname{SE}(\hat{\theta})]$ are the only components needed to compute the SSR [Equation (2)], the variation in development across simulations of the SSR shows the opposite trend with smaller variation for larger true variance values.

Figure 2 shows the development of bias in $S^{2}(\hat{\theta})$ (top row) and bias in SSR (bottom row) with increasing numbers of comparisons per item averaged across all 100 simulations with $68 \%$ confidence intervals for both true variance conditions and all numbers of items. In general, the bias of $S^{2}(\hat{\theta})$ was smaller for larger numbers of items. We first describe the results for a true variance of 0 . The bias of $S^{2}(\hat{\theta})$ was larger for smaller numbers of items, but differences in $S^{2}(\widehat{\theta})$ among numbers of items almost disappeared after about 30 comparisons per item. For 20 and 30 items, the SSR overestimated benchmark reliability in the beginning of data collection. For 20 items, this overestimation stopped after only a few comparisons, but then underestimated benchmark reliability by about 0.2 units, which is a large underestimation. For 30 items, it took about 25 comparisons per item to stop the SSR from overestimating benchmark reliability. For 50 items, the SSR closely estimated benchmark reliability after only a few comparisons per object. For 100 items, the SSR closely estimated benchmark reliability after about 40 comparisons.

We next describe the results for the other true variances. In general, the differences among the number of items conditions in $S^{2}(\hat{\theta})$ were larger for larger true variances. For true variances larger than 1 , on average, $S^{2}(\widehat{\theta})$ was underestimated for 100 items, while it was overestimated for lower numbers of items and lower true variances. Except for a true variance of 3, fewer comparisons were required to converge to the true variance for larger numbers of items. 


\section{STABILITY OF COMPARATIVE JUDGMENT RESULTS}

Interestingly, although it took many comparisons for $S^{2}(\hat{\theta})$ to converge to the true variance, the SSR closely estimated benchmark reliability often after a few comparisons but almost always with 30 comparisons per item. Furthermore, on average, the SSR seemed to closely estimate benchmark reliability after fewer comparisons for lower numbers of items, which is the opposite trend of convergence compared to $S^{2}(\hat{\theta})$. However, the differences in SSR among the numbers of items are quite small in general. One difference worth mentioning is that for 20 items and a true variance of 0.5 , the SSR was overestimated in the beginning of data collection, which is more like the condition with a true variance of zero.

Table 2 shows the mean number of comparisons per item required for accurate $S^{2}(\hat{\theta})$ values. In general, fewer comparisons per item are required on average for larger numbers of items, with the exception of 100 items and a true variance of 3 . In addition, more comparisons per item are required on average for increasing true variances, with the exception of 100 items and a true variance of 3 . The mean number of comparisons per item required for accurate $S^{2}(\hat{\theta})$ values ranges from 24 comparisons per item (for 100 items and a true variance of 1.59) to 119 comparisons per item (for 20 items and a true variance of 0.5 ). Furthermore, the large ranges within each condition indicate that there is a large variation in the number of comparisons per item required across simulations.

Figure 3 shows the development of the coverage of the $95 \%$ confidence intervals for the parameter estimates $\widehat{\boldsymbol{\theta}}$ with increasing numbers of comparisons per item. In general, with the exception of 100 items and a true variance of 3, the coverage was larger than $95 \%$, which indicates that the standard errors of the parameter estimates were overestimated. However, most values are within the range of accurate values. The number of items required for accurate coverage was lower for larger true variances (Figure 3, Table 3). As Table 3 indicates, in many 


\section{STABILITY OF COMPARATIVE JUDGMENT RESULTS}

conditions, the coverage was accurate in 12 comparisons per item or under, and it was accurate for at most 25 comparisons per item.

Because the development of $S^{2}(\hat{\theta})$ and the coverage with increasing number of comparisons per item was different from the development of the SSR, we decided to provide a guideline based on the SSR itself instead of its components. To this end, we computed the number of comparisons per item required for the SSR to underestimate benchmark reliability within a margin in $95 \%$ of the cases. Specifically, we calculated how many comparisons per item were required such that the lower bound of the $95 \%$ CI of the SSR was between the benchmark reliability and a margin of $.10, .05, .03$, and .01 below the benchmark reliability for each condition. The results are displayed in Table 4. The number of comparisons per item required for the SSR to closely estimate benchmark reliability depended on the number of items in the set and the true variance of the item parameters, which is in line with the bottom row in Figures 1 an 2 displaying the SSR in relation to the number of comparisons per item. The number of comparisons per item ranged from 15 to more than 200. In general, smaller margins led to more comparisons per item required, more items in a set led to approximately the same or fewer comparisons per item required, and larger true variances led to fewer comparisons per item required, except for the combination of 20 items and a true variance of 3 .

\section{Discussion}

The guideline that 200 observations per item are required for stable parameter estimates using the Rasch model (Parshall et al., 1998) was adapted for the BTL model in two ways. Guideline 1 was obtained using the number of observations per item in the Rasch model, resulting in 200 comparisons per item for the BTL model. Guideline 2 was obtained using the 


\section{STABILITY OF COMPARATIVE JUDGMENT RESULTS}

total number of observations in a set of items in the Rasch model, resulting in 400 comparisons per item for the BTL model.

In the simulation study, the results showed that the variation in development across simulations of both the estimated variance and the mean squared standard error were larger for larger true variance values, but the variation in development across simulations of the SSR was smaller for larger true variance values. This is interesting, because the estimated variance and the mean squared standard error are the only components of the SSR. Possibly, the variations in the estimated variance and the mean squared standard error are more aligned for larger true variances such that combining them in the SSR leads to less variation. On average, the variance was accurately estimated after 24 to 119 comparisons per item, although the number of comparisons per item differed greatly among simulations. The coverage of the $95 \%$ confidence intervals of the parameter estimates showed that the standard errors of the parameter estimates were accurate after 4 to 25 comparisons per item. The SSR could closely estimate benchmark reliability even when the variance of the parameter estimates was still overestimated. When using margins ranging from .10 to .01 to determine when the SSR closely estimated benchmark reliability, across conditions, the number of comparisons per item ranged from 15 to more than 200.

When we compare the results from the two perspectives, it seems that Guideline 2 of 400 comparisons per item is too pessimistic and overly demanding. Guideline 1 could be useful since several simulations took 200 or more comparisons per item to get stable variance estimates and it took 200 or more comparisons for the SSR to closely estimate benchmark reliability when the margin was .01. However, averaged across samples, the variance was accurately estimated after a maximum of 119 comparisons per item, the standard errors of the parameters and the SSR 


\section{STABILITY OF COMPARATIVE JUDGMENT RESULTS}

required even fewer comparisons per item, and in most conditions, the SSR closely estimated benchmark reliability after less than 50 comparisons per item. Therefore, Guideline 2 may be too demanding as well.

The alternative guideline we present here is largely based on Table 4. We recommend that comparative judgment applications require at least 41 comparisons per item based on the following considerations. With respect to the margin that determines how much the SSR may underestimate benchmark reliability, we are lenient by choosing the largest margin. We believe that this is justified because the benchmark reliability is usually larger than the SSR, and because Verhavert et al. (2019) indicate that the SSR already has high values with this many comparisons per item. If one prefers a smaller margin, we recommend 72 comparisons per item for a margin of .05, 112 comparisons for a margin of .03, and more than 200 comparisons for a margin of .01. With respect to the true variance of the item parameters, we were quite strict by choosing the largest number of comparisons. Because one can never know the true variance in practice and because our study showed that accurate variance estimation often required many observations per item, we argue that it is best to play safe. With respect to the number of items, we also argue to be strict and play safe. However, as one does know the number of items in their comparative judgment application, the required number of comparisons can be somewhat adjusted to the number of items in this set. Table 4 provides information about this adjustment, but the researcher must make the call, given that we only investigated four numbers of items.

Our guideline of 41 comparisons per item renders comparative judgment less interesting to use in practice than the guideline of 12 comparisons per item Verhavert et al. (2019) suggested. However, 41 comparisons per item are necessary for accurately determining the reliability of the measurement using the SSR. The SSR may overestimate benchmark reliability 


\section{STABILITY OF COMPARATIVE JUDGMENT RESULTS}

in individual samples, even when it underestimates reliability on average, especially when the number of comparisons is small. We suggest that after 41 comparisons, the risk of overestimating reliability with the SSR in individual samples is largely reduced.

Our guideline concerns reliability estimation by means of the SSR and not benchmark reliability. This means that using fewer than 41 comparisons may result in sufficient benchmark reliability (Crompvoets et al., 2020, 2021). The problem is that we cannot determine whether this is the case based on the SSR. Therefore, if a different reliability estimate would exist for comparative judgment, the guideline might change. This is an interesting topic for future research.

In our simulation designs, we did not use adaptive pair selection algorithms or multiple raters who perceived a different truth, which are the situations in previous research where the SSR systematically overestimated benchmark reliability. The results of our study provide a baseline how the SSR and the components used to compute the SSR develop with increasing numbers of comparisons when the SSR is expected to underestimate reliability, as it should. Future research could build on our results by investigating how the components of the SSR develop with increasing numbers of comparisons in situations where the SSR might overestimate reliability. The fact that the SSR might overestimate reliability in some situations is even more reason to use a guideline that reduces the risk of overestimation due to sampling fluctuations.

Our study focused on the components of the SSR because we expected that this would show the cause of the inflation of the SSR. However, our simulation study showed that the estimated variance and standard errors of the item parameters developed differently from the SSR with increasing numbers of comparisons with respect to variation between samples, which is not what we expected. Since the components of the SSR developed differently from the SSR, 


\section{STABILITY OF COMPARATIVE JUDGMENT RESULTS}

they do not seem to be the cause of the inflation of the SSR. Future research could also aim at developing alternative reliability estimates to the SSR.

In conclusion, the SSR may overestimate reliability in certain situations, but it can function correctly as an underestimate of reliability even when the variance of the items is overestimated. The SSR can be used when the pairs to be compared are selected without an adaptive algorithm, when raters use the same underlying model/truth, and when the true item variance is at least 1 . The variance of the items is likely to be overestimated when fewer than 24 comparisons per item were performed. An adaptation of the guideline for the Rasch model was too pessimistic. We provided a new guideline of 41 comparisons per item, with nuances concerning the number of items and the margin of accuracy for SSR estimation. Future research is needed to further investigate the SSR estimation and to develop an alternative reliability estimate. 


\section{STABILITY OF COMPARATIVE JUDGMENT RESULTS}

\section{References}

Agresti, A. (1992). Analysis of ordinal paired comparison data. Applied Statistics, 41, 287-297.

Andrich, D. (1978). Relationships between the Thurstone and Rasch approaches to item scaling. Applied Psychological Measurement, 2, 449-460. doi: 10.1177/014662167800200319

Böckenholt, U. (2001). Thresholds and intransitivities in pairwise judgments: A multilevel analysis. Journal of Educational and Behavioral Statistics, 26, 269-282.I

Böckenholt, U. (2006). Thurstonian-based analyses: Past, present, and future utilities. Psychometrika, 71, 615-629. doi: 10.1007/s11336-006-1598-5

Bramley, T. (2015). Investigating the reliability of adaptive comparative judgement. Cambridge Assessment Research Report. Cambridge, United Kingdom: Cambridge Assessment.

Bramley, T. \& Vitello, S. (2018). The effect of adaptivity on the reliability coefficient in adaptive comparative judgement. Assessment in Education: Principles, Policy \& Practice, 2018, 1-16. doi: 10.1080/0969594X.2017.1418734.

Brinkhuis, M. J. S. (2014). Tracking educational progress. (Doctoral dissertation, University of Amsterdam). Retrieved from https://pure.uva.nl/ws/files/2133789/153696_01_1_.pdf.

Cattelan, M. (2012). Models for paired comparison data: A review with emphasis on dependent data. Statistical science, 27, 412-433. doi: 10.1214/12-STS396.

Crompvoets, E. A. V., Béguin, A. A., \& Sijtsma, K. (2020). Adaptive pairwise comparison for educational measurement. Journal of Educational and Behavioral Statistics, 45, 316-338. doi: $10.3102 / 1076998619890589$

Crompvoets, E. A. V., Béguin, A. A., \& Sijtsma, K. (2021). Pairwise comparison using a Bayesian selection algorithm: Efficient holistic measurement. PsyArXiv. https://psyarxiv.com/32nhp/ 


\section{STABILITY OF COMPARATIVE JUDGMENT RESULTS}

Gustafsson, J.-E. (1977). The Rasch model for dichotomous items: Theory, applications and a computer program. Göteborg, Sweden: Göteborg University.

Hunt, T. D., \& Bentler, P. M. (2015). Quantile lower bounds to reliability based on locally optimal splits. Psychometrika, 80, 182-195. doi: 10.1007/s11336-013-9393-6.

Jones, I., \& Alcock, L. (2013). Peer assessment without assessment criteria. Studies in Higher Education, 39, 1774-1787. doi:10.1080/03075079.2013.821974.

Lesterhuis, M., Verhavert, S., Coertjens, L., Donche, V., \& De Mayer, S. (2017). Comparative judgement as a promising alternative to score competences. In: Innovative practices for higher education assessment and measurement. Hershey, PA: IGI Global. doi: 10.4018/978-1-5225-0531-0.ch007.

Lord, F. M. \& Novick, M. R. (1968). Statistical Theories of Mental Test Scores. AddisonWesley.

Luce, R. D. (1959). Individual choice behaviours: A theoretical analysis. New York, NY: Wiley. Maydeu-Olivares, A. (2002). Limited information estimation and testing of Thurstonian models for preference data. Mathematical Social Sciences, 43, 467-483.

Maydeu-Olivares, A. \& Böckenholt, U. (2005). Structural equation modeling of pairedcomparison and ranking data. Psychological Methods, 10, 285-304. doi: 10.1037/1082989X.10.3.285

Newhouse, C. P. (2014). Using digital representations of practical production work for summative assessment. Assessment in Education: Principles, Policy \& Practice, 21, 205220. doi: 10.1080/0969594X.2013.868341. 


\section{STABILITY OF COMPARATIVE JUDGMENT RESULTS}

Parshall, C.G., Davey, T., Spray, J.A. \& Kalohn, J.C. (1998). Computerized Testing-Issues and Applications. A training session presented at the Annual Meeting of the National Council on Measurement in Education.

Pollitt, A. (2012). The method of adaptive comparative judgement. Assessment in Education: Principles, Policy \& Practice, 19, 281-300. doi: 10.1080/0969594X.0962012.0665354.

Rasch, G. (1960). Probabilistic Models for Some Intelligence and Attainment Tests. Danmarks Paedagogiske Institut, Copenhagen.

Sijtsma, K. (2009). On the use, the misuse, and the very limited usefulness of Cronbach's alpha. Psychometrika, 74, 107-120. doi: 10.1007/s11336-008-9101-0.

Stark, S. \& Chernyshenko, O. S. (2011). Computerized adaptive testing with the Zinnes and Griggs pairwise preference ideal point model. International Journal of Testing, 11, 231247. doi: 10.1080/15305058.2011.561459

Thurstone, L. L. (1927). A law of comparative judgment. Psychological Review, 34, 273-86. Van Daal, T., Lesterhuis, M., Coertjens, L., Donche, V., \& De Maeyer, S. (2016). Validity of comparative judgement to assess academic writing: Examining implications of its holistic character and building on a shared consensus. Assessment in Education: Principles, Policy \& Practice, 2016, 1-16. doi: 10.1080/0969594X.2016.1253542.

Van Daal, T. (2020). Making a choice is not easy?!: Unravelling the task difficulty of comparative judgement to assess student work [Doctoral dissertation, University of Antwerp].

Verhavert, S., Bouwer, R., Donche, V., \& De Mayer, S. (2019). A meta-analysis on the reliability of comparative judgement. Assessment in Education: Principles, Policy and Practice, 26, 541-562. doi: 10.1080/0969594X.2019.1602027 


\section{STABILITY OF COMPARATIVE JUDGMENT RESULTS}

Table 1. Total number of observations and number of complete designs according to the translated guideline for the BTL model for different numbers of items.

\begin{tabular}{|c|c|c|c|c|c|c|}
\hline Number of items & 20 & 50 & 100 & 200 & 500 & 1000 \\
\hline \multicolumn{7}{|c|}{ Guideline 1: 200 observations per item } \\
\hline Total number of observations & 2,000 & 5,000 & 10,000 & 20,000 & 50,000 & 100,000 \\
\hline Number of complete designs ${ }^{a}$ & 10.53 & 4.08 & 2.02 & 1.01 & 0.40 & 0.20 \\
\hline \multicolumn{7}{|c|}{ Guideline 2: 400 observations per item } \\
\hline Total number of observations & 4,000 & 10,000 & 20,000 & 40,000 & 100,000 & 200,000 \\
\hline Number of complete designs ${ }^{\mathrm{a}}$ & 21.05 & 8.16 & 4.04 & 2.01 & 0.80 & 0.40 \\
\hline
\end{tabular}

${ }^{\mathrm{a}}$ One complete design contains all $N(N-1) / 2$ unique comparisons. 
STABILITY OF COMPARATIVE JUDGMENT RESULTS

Table 2. Mean number of comparisons per item required for accurate estimation of the true variance.

\begin{tabular}{rcccc}
\hline & \multicolumn{5}{c}{$\begin{array}{c}\text { True variance } \\
M(\text { min-max) }\end{array}$} \\
$\begin{array}{c}\text { Number } \\
\text { of items }\end{array}$ & 0.5 & 1.0 & 1.59 & 3.0 \\
\hline 20 & $119(29-200+)$ & $100(18-200+)$ & $102(21-200+)$ & $88(14-200+)$ \\
30 & $98(18-200+)$ & $78(13-200+)$ & $69(13-200+)$ & $68(13-200+)$ \\
50 & $93(13-200+)$ & $68(14-200+)$ & $50(10-200+)$ & $42(5-161)$ \\
100 & $72(18-200+)$ & $31(4-121)$ & $24(6-94)$ & $54(14-200+)$ \\
\hline
\end{tabular}

a Based on 100 simulations.

Note. The number of comparisons per item represents the average number of comparisons per item in a set of items (i.e., one item may be compared more often than another item) rounded up to integers. 
STABILITY OF COMPARATIVE JUDGMENT RESULTS

Table 3. Mean number of comparisons per item required for accurate coverage of 95\% CI around parameter estimates.

\begin{tabular}{rccccc}
\hline \multirow{5}{*}{$\begin{array}{c}\text { Number } \\
\text { of items }\end{array}$} & 0 & 0.5 & 1.0 & 1.59 & 3.0 \\
\hline 20 & 25 & 4 & 4 & 4 & 5 \\
30 & 9 & 14 & 6 & 6 & 7 \\
50 & 11 & 12 & 12 & 11 & 7 \\
100 & 21 & 21 & 16 & 11 & 4 \\
\hline
\end{tabular}

Note. The number of comparisons per item represents the average number of comparisons per item in a set of items (i.e., one item may be compared more often than another item) rounded up to integers. 
STABILITY OF COMPARATIVE JUDGMENT RESULTS

Table 4. Number of comparisons per item required for the SSR to estimate benchmark reliability between the benchmark reliability value and the benchmark reliability value minus the margin in $95 \%$ of the cases.

\begin{tabular}{|c|c|c|c|c|}
\hline \multirow{2}{*}{$\begin{array}{l}\text { Number } \\
\text { of items }\end{array}$} & \multicolumn{4}{|c|}{ Margin } \\
\hline & .10 & .05 & .03 & .01 \\
\hline & \multicolumn{4}{|c|}{ True variance $=0.5$} \\
\hline 20 & $\underline{41}$ & $\underline{72}$ & $\underline{112}$ & $\underline{200+}$ \\
\hline 30 & $\overline{32}$ & $\overline{62}$ & $\overline{97}$ & $\overline{200+}$ \\
\hline 50 & 32 & 57 & 75 & 175 \\
\hline \multirow[t]{2}{*}{100} & 28 & 45 & 58 & 105 \\
\hline & \multicolumn{4}{|c|}{ True variance $=1$} \\
\hline 20 & 27 & 48 & 70 & 136 \\
\hline 30 & 19 & 33 & 49 & 135 \\
\hline 50 & 18 & 36 & 53 & 108 \\
\hline \multirow[t]{2}{*}{100} & 19 & 30 & 42 & 77 \\
\hline & \multicolumn{4}{|c|}{ True variance $=1.59$} \\
\hline 20 & 23 & 42 & 59 & 119 \\
\hline 30 & 17 & 29 & 42 & 97 \\
\hline 50 & 16 & 25 & 39 & 78 \\
\hline \multirow[t]{2}{*}{100} & 18 & 28 & 37 & 69 \\
\hline & \multicolumn{4}{|c|}{ True variance $=3$} \\
\hline 20 & 25 & 58 & 100 & $\underline{200+}$ \\
\hline 30 & 16 & 27 & 43 & 113 \\
\hline 50 & 15 & 22 & 36 & 83 \\
\hline 100 & 17 & 25 & 33 & 64 \\
\hline
\end{tabular}

Note. The number of comparisons per item represents the average number of comparisons per item in a set of items (i.e., one item may be compared more often than another item) rounded up to integers. Underline for advised (maximum) number of comparisons per item for each threshold. Bold for advised (maximum) number of comparisons per item for each number of items. 